\title{
Erratum to: On the geometry of spaces of oriented geodesics
}

\author{
Dmitri V. Alekseevsky ${ }^{1} \cdot$ Brendan Guilfoyle $^{2}$. \\ Wilhelm Klingenberg ${ }^{3}$
}

Received: 15 March 2016 / Accepted: 11 May 2016 / Published online: 14 June 2016

(C) Springer Science+Business Media Dordrecht 2016

\section{Erratum to: Ann Glob Anal Geom (2011) 40:389-409 DOI 10.1007/s10455-011-9261-5}

We correct some of the entries of Table 1 in our original paper.

1. Those entries of Table 1 in the original paper listing the numbers of geometric structures on the space of geodesics $L^{\epsilon}\left(S^{p, q}\right)$ of the 3-dimensional space $S^{p, q}$ of non-zero constant curvature with $p+q=3$ are incorrect. In this note we give the correct values and arguments. In original paper we failed to note that the $\left(\mathfrak{s o}(V)+\mathfrak{s o}\left(V^{\prime}\right)\right)$-module $V \otimes V^{\prime}$, where $V, V^{\prime}$ are 2-dimensional pseudo-Euclidian spaces, has not only the invariant (para)complex structures $J=J_{V} \otimes 1, J^{\prime}=1 \otimes J_{V^{\prime}}$, but that also their product $J^{\prime \prime}=J J^{\prime}=J_{V} \otimes J_{V^{\prime}}$ is an invariant (para)complex structure. We next analyze the impact of this observation in detail and correct Table 1 accordingly. The authors would like to thank Henri Anciaux for drawing their attention to this oversight.

2. Let $E=E^{p+1, q}$ be a pseudo-Euclidean 4-dimensional vector space of signature ( $p+$ $1, q)=(4,0),(3,1),(2,2)$ or $(1,3)$ with an orthonormal basis $e_{1}, e_{2}, e_{3}, e_{4}$ with $e_{4}^{2}:=$ $g\left(e_{4}, e_{4}\right)=1$. We denote by $E^{\prime}=e_{4}^{\perp}$ the orthogonal complement to $e_{4}$. The pseudo-sphere

The online version of the original article can be found under doi:10.1007/s10455-011-9261-5.

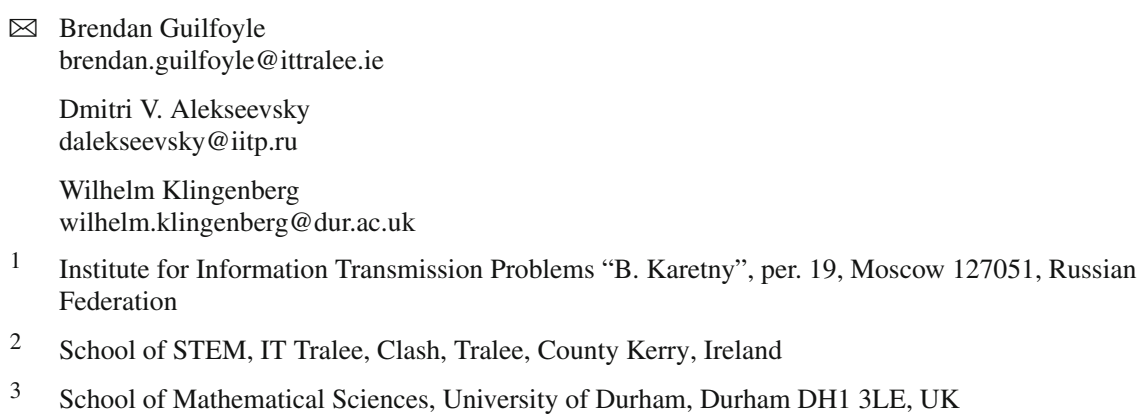


Table 1 Invariant geometric structures

\begin{tabular}{|c|c|c|c|c|c|c|c|c|c|}
\hline & \multirow[t]{2}{*}{ Symplectic structure } & \multicolumn{2}{|c|}{ Complex } & \multicolumn{2}{|c|}{ Para-complex } & \multicolumn{2}{|c|}{ Kähler } & \multicolumn{2}{|c|}{ ParaKähler } \\
\hline & & Int & Non & Int & Non & Int & Non & Int & Non \\
\hline \multicolumn{10}{|l|}{$L^{ \pm}\left(E^{p+1, q}\right)$} \\
\hline$p+1+q \neq 3$ & Symmetric & $\emptyset$ & $\varnothing$ & $\emptyset$ & $\emptyset$ & $\emptyset$ & $\emptyset$ & $\emptyset$ & $\emptyset$ \\
\hline$p+1+q=3$ & & 1 & $\emptyset$ & $\emptyset$ & $\emptyset$ & 1 & $\emptyset$ & $\emptyset$ & $\emptyset$ \\
\hline \multicolumn{10}{|l|}{$L^{+}\left(S^{p, q}\right)$} \\
\hline$p+q>3$ & 1 & 1 & $\varnothing$ & $\emptyset$ & $\emptyset$ & 1 & $\emptyset$ & $\emptyset$ & $\emptyset$ \\
\hline$(p, q)=(3,0)$ & $\mathbb{R}$ & 2 & $\emptyset$ & 1 & $\emptyset$ & $\mathbb{R}, \mathbb{R}$ & $\emptyset$ & $\emptyset$ & $\emptyset$ \\
\hline$(p, q)=(1,2)$ & $\mathbb{R}$ & 2 & $\emptyset$ & 1 & $\varnothing$ & $\mathbb{R}, \mathbb{R}$ & $\emptyset$ & $\emptyset$ & $\emptyset$ \\
\hline$(p, q)=(2,1)$ & $\mathbb{R}$ & 2 & $\emptyset$ & 1 & $\emptyset$ & $\mathbb{R}$ & $\emptyset$ & $\mathbb{R}$ & $\varnothing$ \\
\hline \multicolumn{10}{|l|}{$L^{-}\left(S^{p, q}\right)$} \\
\hline$p+q>3$ & 1 & $\emptyset$ & $\emptyset$ & 1 & $\emptyset$ & $\emptyset$ & $\emptyset$ & 1 & $\emptyset$ \\
\hline$(p, q)=(0,3)$ & $\mathbb{R}$ & 2 & $\emptyset$ & 1 & $\emptyset$ & $\mathbb{R}$ & $\emptyset$ & $\mathbb{R}$ & $\emptyset$ \\
\hline$(p, q)=(2,1)$ & $\mathbb{R}$ & 2 & $\emptyset$ & 1 & $\emptyset$ & $\mathbb{R}$ & $\emptyset$ & $\mathbb{R}$ & $\varnothing$ \\
\hline$(p, q)=(1,2)$ & $\mathbb{R}$ & 2 & $\emptyset$ & 1 & $\emptyset$ & $\emptyset$ & $\emptyset$ & $\mathbb{R}, \mathbb{R}$ & $\emptyset$ \\
\hline$L\left(\mathbb{C} P^{n}\right)$ & $\mathbb{R}$ & 2 & 2 & $\varnothing$ & $\varnothing$ & 2 & 2 & $\emptyset$ & $\emptyset$ \\
\hline$L\left(\mathbb{H} P^{n}\right)$ & 1 & 1 & $\varnothing$ & $\emptyset$ & $\varnothing$ & 1 & $\emptyset$ & $\emptyset$ & $\emptyset$ \\
\hline$L\left(\mathbb{O} P^{2}\right)$ & 1 & 1 & $\varnothing$ & $\emptyset$ & $\emptyset$ & 1 & $\emptyset$ & $\emptyset$ & $\emptyset$ \\
\hline$L\left(\mathbb{C} H^{n}\right)$ & $\mathbb{R}$ & $\emptyset$ & $\varnothing$ & 1 & 1 & $\emptyset$ & $\emptyset$ & 1 & 1 \\
\hline$L\left(\mathbb{H} H^{n}\right)$ & 1 & $\emptyset$ & $\emptyset$ & 1 & 1 & $\emptyset$ & $\emptyset$ & 1 & 1 \\
\hline
\end{tabular}

of unit vectors $S^{p, q}=\left\{x \in E, x^{2}=1\right\}$ is the orbit $S^{p, q}=S O(E) e_{4}$. It has the reductive decomposition

$$
\mathfrak{s o}(E)=\mathfrak{s o}\left(E^{\prime}\right)+e_{4} \wedge E^{\prime},
$$

where $\mathfrak{s o}\left(E^{\prime}\right) \simeq \mathfrak{s o}_{3,0}, \mathfrak{s o}_{2,1}, \mathfrak{s o}_{1,2}, \mathfrak{s o}_{0,3}$, respectively. The metric of $E$ induces an $S O\left(E^{\prime}\right)$ invariant metric in the tangent space $T_{0} S^{p, q}=e_{4} \wedge E^{\prime}$. The stability subalgebra of the geodesic $\ell\left(e_{4} \wedge e_{3}\right)$ through the origin $o=e_{4} \in S^{p, q}$ in the direction $e_{4} \wedge e_{3} \in T_{o} S^{p, q}=$ $e_{4} \wedge E^{\prime}$ is $\mathfrak{s o}(V) \times \mathfrak{s o}\left(V^{\perp}\right)$, where $V=\operatorname{span}\left(e_{1}, e_{2}\right)$ and $V^{\perp}=\operatorname{span}\left(e_{3}, e_{4}\right)$. The geodesic is spacelike if $\epsilon=e_{3}^{2}=1$ and timelike if $\epsilon=e_{3}^{2}=-1$. The space of such geodesics is identified with $L^{\epsilon}\left(S^{p, q}\right)=S O(E) / S O(V) \times S O\left(V^{\perp}\right)$ (up to a covering).

We now denote by $g_{V}$ the unique (up to a scale) metric in 2-dimensional $S O(V)$-module $V$ and by $\omega_{V}$ unique (up to scale ) 2-form. Then for some positive $\lambda$, the complex structure $J_{V}=\lambda g_{V}^{-1} \circ \omega_{V}$ is the unique (up to sign) $\mathfrak{s o}(V)$-invariant complex structure if the metric $g_{V}$ has signature $(2,0)$ or $(0,2)$. It is the unique (up to sign) paracomplex structure if the signature of $g_{V}$ is $(1,1)$. We use similar notations for $V^{\perp}$. The reductive decomposition of the symmetric space $L^{\epsilon}\left(S^{p, q}\right)$ is given by

$$
\mathfrak{s o}(E)=\left(\mathfrak{s o}(V) \oplus\left(\mathfrak{s o}\left(V^{\perp}\right)\right)+V \wedge V^{\perp} .\right.
$$

The tangent space $T_{o} L^{\epsilon}\left(S^{p, q}\right)$ is identified with $\mathfrak{m}:=V \wedge V^{\perp} \simeq V \otimes V^{\perp}$ with the natural action of the isotropy algebra $\mathfrak{h}=\mathfrak{s o}(V)+\mathfrak{s o}\left(V^{\perp}\right)$. We have therefore proved the following. 
Proposition The $\mathfrak{h}$-invariant symmetric bilinear form (respectively, invariant 2-form) on $\mathfrak{m}$ are of the form

$$
h_{\lambda, \mu}=\lambda \omega_{V} \otimes \omega_{V^{\perp}}+\mu g_{V} \otimes g_{V^{\perp}}, \quad \omega_{\lambda, \mu}=\lambda g_{V} \otimes \omega_{V^{\perp}}+\mu \omega_{V} \otimes g_{V^{\perp}}
$$

for $\lambda, \mu \in \mathbb{R}$.

The endomorphisms $J:=J_{V} \otimes 1, J^{\perp}:=1 \otimes J_{V^{\perp}}, J^{\prime}:=J \circ J^{\perp}=J_{V} \otimes J_{V^{\perp}}$ are invariant (para)complex structure in $\mathrm{m}$. Moreover, $J . J^{\perp}$ are skew-symmetric with respect to any metric $h_{\lambda, \mu}$ and the endomorphism $J^{\prime}$ is symmetric.

Since $S^{p, q}$ is an irreducible symmetric space, any invariant tensor field is parallel. We obtain

Corollary Any non-degenerate form $h_{\lambda, \mu}$ defines an invariant pseudo-Riemannian metric, any non-degenerate 2-form $\omega_{\lambda, \mu}$ defines an invariant symplectic form and the endomorphisms $J, J^{\perp}, J^{\prime}$ define integrable (para)complex structures on $L^{\epsilon}\left(S^{p, q}\right)$. Furthermore, any pair of the form $\left(h_{\lambda, \mu}, J\right)$ or $\left(h_{\lambda . \mu}, J^{\perp}\right)$, where $h_{\lambda, \mu}$ is as above, defines a (para)Kähler structure on $\mathfrak{m}$.

3. In the following table we indicate the signature of the metrics $g_{V}$ and $g_{V \perp}$ for different values of $p$ and $\epsilon$ and the type of the endomorphisms $J=J_{V} \otimes 1, J^{\perp}=1 \otimes J_{V^{\perp}}, J^{\prime}=$ $J_{V} \otimes J_{V} \perp(\mathrm{cx}=$ complex structure, para $=$ paracomplex structure $)$. Note also that there is

\begin{tabular}{lllllll}
\hline $\mathrm{p}$ & $\epsilon$ & $g_{V}$ & $g_{V \perp}$ & $J$ & $J^{\perp}$ & $J^{\prime}$ \\
\hline 3 & + & $(2,0)$ & $(2,0)$ & $\mathrm{cx}$ & $\mathrm{cx}$ & para \\
2 & + & $(1,1)$ & $(2,0)$ & para & $\mathrm{cx}$ & $\mathrm{cx}$ \\
2 & - & $(2,0)$ & $(1,1)$ & $\mathrm{cx}$ & para & $\mathrm{cx}$ \\
1 & + & $(0,2)$ & $(2,0)$ & $\mathrm{cx}$ & $\mathrm{cx}$ & para \\
1 & - & $(1,1)$ & $(1,1)$ & para & para & $\mathrm{cx}$ \\
0 & - & $(0,2)$ & $(1,1)$ & $\mathrm{cx}$ & para & $\mathrm{cx}$ \\
\hline
\end{tabular}

a natural isomorphism $L^{ \pm}\left(S^{p, q}=L^{\mp}\left(S_{-}^{q, p}\right)\right.$, where $S_{-}^{q, p}=\left\{x \in E^{q, p+1}, x^{2}=-1\right\}$. We finally reproduce Table 1 in its corrected form. 